\title{
La dieta mediterránea resulta beneficiosa en la prevención primaria de eventos cardiovasculares
}

\author{
Mediterranean Diet show benefits on primary prevention of cardiovascular disease
}

Estruch R y col. NEJM. 2013;368(14):1279-90.

\section{Objetivos}

Evaluar el impacto de la Dieta Mediterránea (DM) en la reducción de muerte por enfermedad cardiovascular (ECV), del accidente cerebrovascular (ACV) e infarto de miocardio (IAM) en una población de alto riesgo cardiovascular.

\section{Diseño y población}

Ensayo clínico aleatorizado y controlado multicéntrico, realizado en España. Incluyó personas con alto riesgo cardiovascular pero sin enfermedad cardiovascular al momento del enrolamiento. Tenía tres ramas de intervención: DM con aceite de oliva, DM con frutas secas y una dieta control baja en calorías.

\section{Descripción del seguimiento}

Todos los participantes debían asistir, cada tres meses, a una entrevista individual motivacional y a sesiones grupales, de acuerdo a la rama de intervención, donde se daba información oral y escrita sobre los objetivos y características de las dietas y recomendaciones sobre cómo elaborarlas. A esto se agregaba la administración de aceite de oliva y frutas secas para cada una de las $\mathrm{DM}, \mathrm{y}$ de regalos no comestibles para la dieta control.
Vale aclarar que en la rama control estas intervenciones se realizaron anualmente los primeros tres años del ensayo, continuando igual al resto de los grupos para el restante del seguimiento. Para evaluar la adherencia a la dieta con aceite de oliva y con frutas secas se tomaron al azar participantes de las tres ramas y se dosaron dos metabolitos bioquímicos al primer, tercer y quinto año.

\section{Medición de resultados}

El resultado primario fue el número combinado de ECV. Los resultados secundarios fueron la incidencia de ACV, IAM, muerte por ECV y muerte de causa no cardiovascular. Los profesionales de la salud que constataban los eventos se encontraban cegados a la intervención recibida. El análisis primario fue por intención de tratar.

\section{Resultados principales}

Un total de 7.442 personas se siguieron por una mediana de 4,8 años. En total ocurrieron 288 eventos primarios: 96 en el grupo con aceite de oliva, 83 en el grupo de frutas secas y 109 en la dieta control. Los principales resultados se resumen en la tabla 1. Los resultados secundarios no mostraron diferencias

Tabla 1: Resultados principales de acuerdo al grupo de estudio.

\begin{tabular}{|c|c|c|c|c|c|}
\hline Resultado & $\begin{array}{l}\text { DM aceite oliva } \\
(\mathrm{N}=2.543)\end{array}$ & $\begin{array}{l}\text { DM frutos secos } \\
(\mathrm{N}=2.454)\end{array}$ & $\begin{array}{c}\text { Dieta control } \\
(\mathrm{N}=2.450)\end{array}$ & $\begin{array}{l}\text { DM aceite oliva vs } \\
\text { dieta control }\end{array}$ & $\begin{array}{l}\text { DM frutos secos vs } \\
\text { dieta control }\end{array}$ \\
\hline & \multicolumn{3}{|c|}{ N/1000 personas/año (IC 95\%) } & \multicolumn{2}{|c|}{$\mathbf{p}$} \\
\hline ECV combinados ${ }^{\dagger}$ & $8,1(6,6$ a 9,9$)$ & $8,0(6,4$ a 9,9$)$ & $11,2(9,2$ a 13,5$)$ & 0,009 & 0,02 \\
\hline \multirow[t]{2}{*}{ ACV } & $4,1(3,1$ a 5,5$)$ & $3,1(2,1$ a 4,4$)$ & $5,9(4,5$ a 7,7$)$ & 0,03 & 0,003 \\
\hline & \multicolumn{3}{|c|}{ Hazard ratio para cada DM vs control (IC 95\%) } & \multicolumn{2}{|c|}{$\mathbf{p}$} \\
\hline 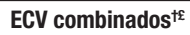 & $0,70(0,54$ a 0,92$)$ & $0,72(0,54$ a 0,96$)$ & 1,00 (ref) & 0,01 & 0,03 \\
\hline ACV $\mathbf{E}$ & $0,67(0,46$ a 0,98$)$ & $0,54(0,35$ a 0,84$)$ & 1,00 (ref) & 0,04 & 0,006 \\
\hline
\end{tabular}

DM: dieta mediterránea. ECV: eventos cardiovasculares. ACV: accidente cerebrovascular.

$†$ †ccidente cerebrovascular, Infarto de miocardio, y muerte por enfermedad cardiovascular. £Ajustados por sexo, edad, antecedentes familiares de enfermedad coronaria precoz, tabaquismo, índice de masa corporal, hipertensión arterial, dislipemia y diabetes.

significativas, salvo por la incidencia de ACV.

Conclusión

La DM enriquecida en aceite de oliva y frutas secas demostró reducir los eventos cardiovasculares, en especial el ACV, en individuos con alto riesgo cardiovascular libres de enfermedad. Fuente de financiamiento y conflictos de interés de los autores: Financiación de organismos públicos.

\section{Comentario}

Si bien las tres dietas eran muy similares entre sí, las DM se diferenciaban de la dieta control por el alto consumo de aceite de oliva y frutas secas, y por la fuerte recomendación de consumir pescados y legumbres.

Se ha demostrado por estudios previos la relación beneficiosa entre la DM y la prevención secundaria de $\mathrm{ECV}^{1-3}$. Este estudio evidencia la prevención primaria de ECV en personas de alto riesgo cardiovascular. Sus conclusiones, no obstante, deben aplicarse a este grupo de riesgo, sin poder extrapolarse a personas de mediano y bajo riesgo.

\section{Conclusiones del comentador}

Se deberían realizar más estudios comparativos entre estos alimentos y, por qué no, compararlos con el uso de estatinas, siendo estos uno de los fármacos más usados en prevención primaria de ECV.

Natividad Burdisso [ Servicio de Medicina Familiar y Comunitaria del Hospital Italiano de Buenos Aires. natividad.burdisso@ hospitalitaliano.org.ar ]

Burdisso, N. La dieta mediterránea resulta beneficiosa en la prevención primaria de eventos cardiovasculares. Evid Act Pract Ambul. Ene-Mar 2014;17(1):10. Comentado de: Estruch R et al. Primary Prevention of Cardiovascular Disease with a Mediterranean Diet. NEJM. 2013; 368(14):1279-90. PMID: 23432189.

\section{Referencias}

1.Sofi F, Abbate R, Gensini GF, Casini. A. Accruing evidence on benefits of adherence to the Mediterranean diet on health: an updated systematic review and meta-analysis. Am J Clin Nutr 2010;92:1189-96.

2. Serra-Majem L, Roman B, Estruch R. Scientific evidence of interventions using the Mediterranean diet: a systematic review. Nutr Rev 2006;64:S27-S47.

3. de Lorgeril M, Salen P, Martin JL, Monjaud I, Delaye J, Mamelle N. Mediterranean diet, traditional risk factors, and the rate of cardiovascular complications after myocardial infarction: final report of the Lyon Diet Heart Study. Circulation 1999; 99:779-85. 